\title{
Determinants of Medication Adherence for Pulmonary Tuberculosis Patients During Continuation Phase in Dalian, Northeast China
}

This article was published in the following Dove Press journal: Patient Preference and Adherence

\author{
Liang Du (D) \\ Xu Chen' \\ Xuexue Zhu' \\ Yu Zhang' \\ Ruiheng $\mathrm{Wu}^{\prime}$ \\ Jia $\mathrm{Xu}{ }^{\prime}$ \\ Haoqiang $\mathrm{Ji}^{1}$ \\ Ling Zhou' \\ Xiwei $\mathrm{Lu}^{2}$
}

'School of Public Health, Dalian Medical University, Dalian, Liaoning II6044, People's Republic of China; ${ }^{2}$ Department of Tuberculosis Internal Medicine, Dalian Tuberculosis Hospital, Dalian, Liaoning I I603I, People's Republic of China
Correspondence: Ling Zhou; Xiwei Lu

Tel +86-4I I-86I I-0368

Email zhouling0609@।63.com;

tb_luxiwei@I26.com
Purpose: Medication adherence is crucial for decreasing the burden of tuberculosis, but few relevant studies have been conducted in northeast China. This study aimed to explore the level of medication adherence among pulmonary tuberculosis outpatients and the predictive factors based on the bio-psycho-social medical model.

Patients and Methods: A cross-sectional multi-center survey was conducted in four tuberculosis medical institutions in Dalian, northeast China. Medication adherence was measured using the eight-item Chinese version of the Morisky Medication Adherence Scale, which divides adherence into three levels. The independent variables consisted of sociodemographic characteristics, treatment factors, knowledge about TB, mental health, and behavioral characteristics. Descriptive statistics, the chi-square test, and multivariate ordinal logistic regression were applied to analyze the data using Stata/MP 14.0.

Results: Among the 564 eligible participants, 236 (41.84\%) and 183 (32.45\%) exhibited high and medium medication adherence, respectively, but 145 (25.71\%) exhibited low medication adherence. Multivariate ordinal logistic regression showed that patients who were older (OR: 1.02, $p=0.013$ ) were employed (OR: 1.61, $p=0.011)$, had better tuberculosis knowledge (OR: 1.34, $p<0.001$ ), and did not consume alcohol (OR: 1.84, $p=0.032$ ) exhibited higher medication adherence. However, patients who did not follow their doctors' advice to take adjuvant drugs (OR: $0.44, p=0.001$ ), had a history of TB treatment (OR: 1.76, $\mathrm{p}=0.009$ ), experienced adverse drug reactions (OR: $0.65, p=0.017$ ), experienced stigma (OR: 0.67 , $p=0.032$ ), and needed supervised treatment (OR: $0.66, p=0.012)$ exhibited lower medication adherence.

Conclusion: Tuberculosis patients' medication adherence was not very high and it was influenced by diverse and complex factors involving sociodemographic characteristics, treatment factors, knowledge about TB, mental health, and behavioral characteristics.

Keywords: tuberculosis patients, treatment, influence factors, bio-psycho-social medical model, Chinese context

\section{Introduction}

Tuberculosis (TB) is a widespread infectious disease caused by Mycobacterium tuberculosis (MTB). According to the World Health Organization (WHO), there were about 10.0 million new TB cases (range, 9.0-11.1 million), equivalent to 133 cases (range, 120-148) per 100 000, worldwide in $2018 .^{1} \mathrm{~TB}$ is also one of the top 10 causes of death globally and it caused an estimated 1.2 million (range, 1.1-1.3 million) deaths in 2018. ${ }^{1}$ Although free anti-TB medicines (including isoniazid, rifampicin, ethambutol, streptomycin, pyrazinamide and so on) have 
been provided free of charge by the government, China has now the second-highest TB burden in the world, accounting for about $9 \%$ of cases, following India (27\%). ${ }^{1}$ It showed high morbidity and mortality of TB in northeast China according to the China Health Statistics Yearbook in 2019. The global End TB strategy is difficult to carry out in the current Chinese context, but medication adherence is a crucial issue related to TB prevention and control. $^{2,4}$

The TB patients have to require typically at least 6 months of treatment regimen. After an intensive phase treatment for 2 months with a four-drug regimen (isoniazid, rifampicin, pyrazinamide, ethambutol), the TB patients have to accept the continuation phase lasting more than 4 months according to the WHO recommendation. TB patients could be more likely to show nonadherence in continuation phase because they have improved signs and symptoms of the disease and even might think they are cured; thus, they might be careless in taking medications. ${ }^{5,6}$ Poor anti-TB medication adherence not only causes disease deterioration, relapse, and even drug resistance but also the spread of MTB to others, which increases the burden related to TB control. ${ }^{4,7}$ However, previous studies demonstrated that the medication adherence of Chinese TB patients under the directly observed treatment short-course (DOTS) strategy recommended by the WHO was not very perfect, especially in economically disadvantaged areas and among migrant patients. A survey in five provinces of central and western China found that about $20 \%$ of TB patients did not adhere to regular treatment. ${ }^{8}$ Over a third of TB patients exhibited low medication adherence according to another study conducted in west China. ${ }^{9}$ A study conducted in 12 counties in Shandong province in east China indicated that migrant TB patients exhibited inferior medication adherence. ${ }^{10} \mathrm{~TB}$ patients' medication adherence is clearly not very high in a certain population of China, and there is a lack of research on this topic in northeast China.

Many quantitative studies have explored the factors associated with medication adherence among TB patients in the past decades. A cross-sectional questionnaire-based study conducted in Russia found that social and psychological factors were significantly associated with medication adherence among TB patients. ${ }^{11}$ Another study conducted in Sri Lanka found that treatment-related adverse events, such as experiencing side effects of anti-TB drugs, were associated with low medication adherence. ${ }^{12}$ Additionally, knowledge about TB treatment and mental health (such as anxiety and stigma) have been shown to be important determinants of medication adherence. ${ }^{13,15}$ Moreover, a previous study has found that unhealthy habits such as smoking and alcohol consumption are also associated with low medication adherence. ${ }^{16}$ However, previous studies in China only explored the determinants of TB patients' medication adherence by assessing sociodemographic factors, ${ }^{9}$ TB knowledge and cognition, ${ }^{17}$ medication supervision mode and social support, ${ }^{10}$ with a lack of research on treatment factors and stigma.

TB patients' medication adherence should not only be considered as a clinical issue but also a public health problem. The bio-psycho-social medical model emphasizes that psychological and social factors that influence human health promotion should be considered in addition to biological factors, in contrast to the traditional medical model that emphasizes biological determinants. ${ }^{18,19}$ Based on this model, the purpose of this study was to explore the level of TB patients' medication adherence in northeast China during the continuation phase, in which patients tend to be more likely to exhibit poor adherence, ${ }^{20}$ and to analyze the determinants of medication adherence in terms of sociodemographic characteristics, treatment factors, knowledge about TB, mental health, and behavioral characteristics.

\section{Patients and Methods Study Design and Setting}

This cross-sectional multi-center survey was conducted between June 20, 2019 and August 31, 2019. Four medical institutions in Dalian, Liaoning province, in northeast China were involved. The four medical institutions, which served different types of TB patients, were chosen according to their institution level and location. The first medical institution was the Dalian TB hospital, which mainly serves TB patients across the whole city, especially critically ill and urban patients. The second was Pulandian TB hospital, which is a branch hospital of the first hospital and it serves both rural and urban TB patients. The other two medical institutions were Zhuanghe and Lvshun TB dispensaries, which only serve local patients with milder TB. Outpatients with pulmonary TB who had been taking anti-TB medicine for $>2$ months were eligible. We excluded patients aged $<15$ years and patients who were unable to complete the questionnaire due to unconsciousness. Beside the members of our research team, we recruited 15 interviewers from the School of Public 
Health at Dalian Medical University. We trained the interviewers before conducting the formal face-to-face interview, and they were responsible for filling in the questionnaire.

\section{Questionnaire}

The questionnaire was designed based on a literature review and expert consultation. It mainly collected data on medication adherence, sociodemographic characteristics, treatment factors, knowledge about TB, mental health, and behavioral characteristics. Medication adherence was assessed using the eight-item Morisky Medication Adherence Scale (MMAS-8), ${ }^{21}$ which is a process-oriented way to measure medication adherence. $^{22}$ The scale has acceptable reliability and validity, and Cronbach's $\alpha$ was 0.74 in the current study. Each item represents a score of 1 , and the maximum score is 8 . Medication adherence was divided into three levels according to previous studies (8: high adherence; 6 to $<8$ : medium adherence; and <6: low adherence). ${ }^{23,24}$

Based on the bio-psycho-social medical model, factors that potentially influenced medication adherence were divided into four aspects. First, we took sociodemographic characteristics into consideration, comprising patients' gender, age, employment status, migration status, residence (urban or rural), and education level. Second, we assessed treatment factor, comprising a previous history of TB treatment, medication duration (from the beginning of treatment to the survey date), number of anti-TB medicines, taking only free anti-TB medicine, adjuvant drug usage (such as hepatinica) and adverse drug reactions. Third, we considered participants' knowledge about TB and mental health (anxiety and stigma). We used six commonsense questions on Chinese TB prevention and control practices to assess TB knowledge, such as "What do you think the main route of TB transmission is?" and "What do you think irregular antiTB medication usage will bring about?" Each question had four options and each correct answer was worth one point. We also recorded self-assessed TB severity. Anxiety was measured using the 7-item Generalized Anxiety Disorder scale (GAD-7) ${ }^{25}$ and divided into four levels (minimal, mild, moderate, and severe); ${ }^{25}$ Cronbach's $\alpha$ was 0.95 in the current study. Perceived stigma was measured using a 9-item stigma questionnaire developed according to the Chinese sociocultural context ${ }^{26}$ and divided into a binary variable (Yes/No) based on the median score ${ }^{26}$ Cronbach's $\alpha$ was 0.89 in the current study. Fourth, we assessed behavioral characteristics, comprising smoking, alcohol consumption, and treatment supervision (need or no need for supervision from family, doctors, or others when taking anti-TB medicine).

\section{Statistical Analysis}

A sample of 593 records containing the questionnaire data was collected, and we authors used EpiData software version 3.1 (EpiData Association, Odense, Denmark) to set up a database to enter the questionnaire data. We deleted records if they contained $>20 \%$ missing values, leading to 564 eligible records in the final sample. The categorical variables are described using numbers and percentages, and the continuous variables are described using mean and median. Intergroup comparisons were performed using the chi-square test for categorical variables. Ordinal multivariate logistic regression was performed to determine the predictors of TB patients' medication adherence; odds ratios (ORs) with 95\% confidence intervals (CIs) were calculated. To test as many variables as possible, we introduced variables with $p$-value $<0.25$ in the chisquare tests into the multivariate regression model. The level of statistical significance was $p$-value $<0.05$. All statistical analyses were performed using Stata/MP version 14.0 (StataCorp, College Station, TX, USA).

\section{Ethics Approval and Informed Consent}

The study protocol was reviewed and approved by the ethics committee of Dalian Medical University, Liaoning province, China. The ethics committee of Dalian Medical University also approved participants under the age of 18 years providing written informed consent on their own behalf. Prior to being interviewed by staff from the School of Public Health at Dalian Medical University (who filled in the questionnaires), participants received verbal and written information about the purpose and structure of the interview, and they signed an informed consent form to participate in our study. For the participants under the age of 18 years, we ask for the permission from them and their parents before the patients wrote the informed consent.

\section{Results}

\section{Sociodemographic Characteristics}

Among the 564 participants, 236 (41.84\%) and 183 (32.45\%) exhibited high and medium medication adherence, respectively, but 145 (25.71\%) exhibited low medication adherence. There were about twice as many male patients 
$(66.31 \%)$ as female patients $(33.69 \%)$. The mean age was 47.41 years and the median was 49 years (interquartile range 31 years). Most patients were employed (71.63\%) even though they were still in the TB treatment phase, but 160 (28.37\%) were unemployed. Only 93 (16.49\%) were migrants, and there were slightly more rural patients $(52.66 \%)$ than urban patients $(47.34 \%)$. Middle school education $(34.22 \%)$ was the most common education level, and the percentages among the other education levels were almost the same as each other. The chi-square tests indicated that unemployment and education were associated with medication adherence $(p<0.05)$ (Table 1$)$.

\section{Treatment Factors}

Over $80 \%$ of participants were new TB patients, and only $107(18.97 \%)$ had a history of TB treatment. The mean medication duration was 7.87 months and the median was 6 months; $332(58.87 \%)$ patients had a medication duration of 2-6 months, and 91 (16.13\%) had a medication duration of $>12$ months. The median number of anti-TB medicines was three, and $48.94 \%$ of the patients took $>3$ medicines. About one-fifth only took free anti-TB medicine and did not purchase extra anti-TB drugs. Most of the patients $(87.59 \%)$ took adjuvant medicine (such as hepatinica) according to their doctors' advice. There were 199 (35.28\%) patients who reported having adverse drug effects such as nausea, stomach discomfort, and liver pain. The chi-square tests indicated that having a history of TB treatment, taking adjuvant drugs and experiencing adverse effects were associated with medication adherence $(p<0.05)$ (Table 2$)$.

\section{TB Knowledge and Mental Health Status}

The mean TB knowledge score was 4.58 and the median was 5. Many patients $(61.35 \%)$ had a good knowledge of TB prevention and treatment (score, 5-6), but 44 (7.80\%) had a score $<3$. Among the participants, $386(68.44 \%)$ had no or mild anxiety, but approximately $6 \%$ reported moderate or severe anxiety during TB treatment. The mean stigma score was 18.45 and the median was 19; 247 (43.79\%) patients reported self-perceived stigma. Many participants reported that their disease was not very serious (41.31\%); 140 $(24.82 \%)$ thought their disease not serious at all; but about one-third thought their disease was moderate to very serious. The chi-square tests indicated that anxiety, stigma, and knowledge about TB were all significantly associated with medication adherence $(p<0.05)$ (Table 3$)$.

\section{Behavioral Characteristics}

Most participants did not smoke while in the treatment period, but there were still $88(15.60 \%)$ patients who could not quit smoking. Patients who did not consume alcohol $(89.89 \%)$ accounted for the vast majority, but 57 (10.11\%)

Table I Medication Adherence Level by Demographic and Social Characteristics

\begin{tabular}{|c|c|c|c|c|c|c|}
\hline \multirow[t]{2}{*}{ Variable } & \multirow[t]{2}{*}{ Description } & \multirow[t]{2}{*}{$\mathbf{N}(\%)$} & \multicolumn{3}{|c|}{ Adherence Level } & \multirow[t]{2}{*}{ Chi-Square Test $p$-value } \\
\hline & & & Low & Medium & High & \\
\hline \multirow[t]{2}{*}{ Gender } & Male & $374(66.3 \mathrm{I})$ & 91 & 119 & 164 & \multirow[t]{2}{*}{0.364} \\
\hline & Female & $190(33.69)$ & 54 & 64 & 72 & \\
\hline \multirow[t]{4}{*}{ Age (years) } & $<21$ & $26(4.61)$ & 11 & 11 & 4 & \multirow[t]{4}{*}{0.093} \\
\hline & $21-40$ & $196(34.75)$ & 51 & 61 & 84 & \\
\hline & $4 I-60$ & 181 (32.09) & 38 & 63 & 80 & \\
\hline & $>60$ & $161(28.55)$ & 45 & 48 & 68 & \\
\hline \multirow[t]{2}{*}{ Unemployment } & Yes & $160(28.37)$ & 49 & 61 & 50 & \multirow[t]{2}{*}{0.006} \\
\hline & No & $404(71.63)$ & 96 & 122 & 186 & \\
\hline \multirow[t]{2}{*}{ Immigration } & Yes & 93 (16.49) & 27 & 31 & 35 & \multirow[t]{2}{*}{0.614} \\
\hline & No & $47 \mid(83.5 I)$ & 118 & 152 & 201 & \\
\hline \multirow[t]{2}{*}{ Residence } & Urban & $267(47.34)$ & 71 & 79 & 117 & \multirow[t]{2}{*}{0.386} \\
\hline & Rural & $297(52.66)$ & 74 & 104 & 119 & \\
\hline \multirow[t]{4}{*}{ Education } & Primary or below & $123(21.28)$ & 40 & 39 & 44 & \multirow[t]{4}{*}{0.031} \\
\hline & Middle school & $193(34.22)$ & 42 & 69 & 82 & \\
\hline & High school & $120(21.28)$ & 33 & 26 & 61 & \\
\hline & College or above & $128(22.70)$ & 30 & 49 & 49 & \\
\hline
\end{tabular}


Table 2 Medication Adherence Level by TB Patients' Treatment Factors

\begin{tabular}{|c|c|c|c|c|c|c|}
\hline \multirow[t]{2}{*}{ Variable } & \multirow[t]{2}{*}{ Description } & \multirow[t]{2}{*}{$\mathbf{N}(\%)$} & \multicolumn{3}{|c|}{ Adherence Level } & \multirow[t]{2}{*}{ Chi-Square Test $p$-value } \\
\hline & & & Low & Medium & High & \\
\hline History of TB treatment & $\begin{array}{l}\text { Yes } \\
\text { No }\end{array}$ & $\begin{array}{l}107(18.97) \\
457(81.03)\end{array}$ & $\begin{array}{l}44 \\
101\end{array}$ & $\begin{array}{l}30 \\
153\end{array}$ & $\begin{array}{l}33 \\
203\end{array}$ & $<0.001$ \\
\hline Medication duration (months) & $\begin{array}{l}2-6 \\
7-9 \\
10-12 \\
>12\end{array}$ & $\begin{array}{l}332(58.87) \\
84(14.89) \\
57(10.11) \\
91(16.13)\end{array}$ & $\begin{array}{l}80 \\
17 \\
20 \\
28\end{array}$ & $\begin{array}{l}110 \\
28 \\
11 \\
34\end{array}$ & $\begin{array}{l}142 \\
39 \\
26 \\
29\end{array}$ & 0.095 \\
\hline Number of anti-TB medicines & $\begin{array}{l}\leq 3 \\
>3\end{array}$ & $\begin{array}{l}288(51.06) \\
276(48.94)\end{array}$ & $\begin{array}{l}78 \\
67\end{array}$ & $\begin{array}{l}80 \\
103\end{array}$ & $\begin{array}{l}130 \\
106\end{array}$ & 0.052 \\
\hline Free anti-TB medicine only & $\begin{array}{l}\text { Yes } \\
\text { No }\end{array}$ & $\begin{array}{l}\text { II } 13(20.04) \\
451(79.96)\end{array}$ & $\begin{array}{l}30 \\
115\end{array}$ & $\begin{array}{l}40 \\
143\end{array}$ & $\begin{array}{l}43 \\
193\end{array}$ & 0.637 \\
\hline Adjuvant drug use & $\begin{array}{l}\text { Yes } \\
\text { No }\end{array}$ & $\begin{array}{l}494 \text { (87.59) } \\
70(12.4 I)\end{array}$ & $\begin{array}{l}121 \\
24\end{array}$ & $\begin{array}{l}153 \\
30\end{array}$ & $\begin{array}{l}220 \\
16\end{array}$ & 0.003 \\
\hline Adverse drug reaction & $\begin{array}{l}\text { Yes } \\
\text { No }\end{array}$ & $\begin{array}{l}199(35.28) \\
365(64.72)\end{array}$ & $\begin{array}{l}63 \\
82\end{array}$ & $\begin{array}{l}70 \\
113\end{array}$ & $\begin{array}{l}66 \\
170\end{array}$ & 0.005 \\
\hline
\end{tabular}

Abbreviation: TB, tuberculosis.

reported that they consumed alcohol while in treatment. Over half of the patients reported unsupervised treatment, taking their anti-TB medicine on their own. However, 239 $(42.38 \%)$ could not do this and needed family, doctors, or others to remind them to take their anti-TB medicine. The chi-square test indicated that alcohol consumption was associated with medication adherence $(p<0.05)$ (Table 4$)$.
Determinants of Patients' Medication Adherence Based on Ordinal Logistic Regression

The ordinal logistic regression results showed that increasing age led to an increase in medication adherence $(p=0.013)$. In addition, medication adherence increased with employment (OR: 1.61, 95\% CI: 1.12-2.32, $p=0.011$ ), knowledge about TB

Table 3 Medication Adherence Level by TB Patients' Knowledge About TB and Mental Health

\begin{tabular}{|c|c|c|c|c|c|c|}
\hline \multirow[t]{2}{*}{ Variable } & \multirow[t]{2}{*}{ Description } & \multirow[t]{2}{*}{$\mathbf{N}(\%)$} & \multicolumn{3}{|c|}{ Adherence Level } & \multirow[t]{2}{*}{ Chi-Square Test $p$-value } \\
\hline & & & Low & Medium & High & \\
\hline \multirow[t]{3}{*}{ Knowledge about TB } & $\mathrm{I}-2$ & $44(7.80)$ & 21 & 14 & 9 & \multirow[t]{3}{*}{0.001} \\
\hline & $3-4$ & $174(30.85)$ & 51 & 58 & 65 & \\
\hline & $5-6$ & $346(61.35)$ & 73 & 111 & 162 & \\
\hline \multirow[t]{4}{*}{ Anxiety } & Minimal & $386(68.44)$ & 94 & 108 & 184 & \multirow[t]{4}{*}{$<0.001$} \\
\hline & Mild & $|4|(25.00)$ & 36 & 65 & 40 & \\
\hline & Moderate & $26(4.6 I)$ & 9 & 8 & 9 & \\
\hline & Severe & II (1.95) & 6 & 2 & 3 & \\
\hline \multirow[t]{2}{*}{ Stigma } & No & $317(56.21)$ & 62 & 103 & 152 & \multirow[t]{2}{*}{$<0.001$} \\
\hline & Yes & 247 (43.79) & 83 & 80 & 84 & \\
\hline \multirow[t]{5}{*}{ Self-assessed severity } & Very serious & $12(2.13)$ & 4 & 4 & 4 & \multirow[t]{5}{*}{0.065} \\
\hline & Serious & $60(10.64)$ & 21 & 22 & 17 & \\
\hline & Moderately serious & $119(21.10)$ & 39 & 39 & 41 & \\
\hline & Not very serious & $233(4 \mid .3 I)$ & 47 & 77 & 109 & \\
\hline & Not serious at all & $140(24.82)$ & 34 & 41 & 65 & \\
\hline
\end{tabular}

Abbreviation: TB, tuberculosis. 
Table 4 Medication Adherence Level by TB Patients' Behavioral Characteristics

\begin{tabular}{|c|c|c|c|c|c|c|}
\hline \multirow[t]{2}{*}{ Variable } & \multirow[t]{2}{*}{ Description } & \multirow[t]{2}{*}{$\mathbf{N}(\%)$} & \multicolumn{3}{|c|}{ Adherence Level } & \multirow[t]{2}{*}{ Chi-Square Test $p$-value } \\
\hline & & & Low & Medium & High & \\
\hline \multirow[t]{2}{*}{ Smoking } & Yes & $88(15.60)$ & 29 & 30 & 29 & \multirow[t]{2}{*}{0.123} \\
\hline & No & $476(84.40)$ & 116 & 153 & 207 & \\
\hline \multirow[t]{2}{*}{ Alcohol consumption } & Yes & $57(10.11)$ & 20 & 22 & 15 & \multirow[t]{2}{*}{0.038} \\
\hline & No & $507(89.89)$ & 125 & 161 & 221 & \\
\hline \multirow[t]{2}{*}{ Medication supervision } & Unsupervised & $325(57.62)$ & 75 & 107 & 143 & \multirow[t]{2}{*}{0.226} \\
\hline & Supervised & $239(42.38)$ & 70 & 76 & 93 & \\
\hline
\end{tabular}

Abbreviation: TB, tuberculosis.

prevention and treatment (OR: 1.34, 95\% CI: 1.16-1.54, $p<0.001$ ), and lack of alcohol consumption (OR: 1.84, 95\% CI: $1.05-3.22, p=0.032$ ) during the treatment period. In contrast, those who did not follow their doctors' advice regarding taking adjuvant drugs (OR: $0.44,95 \%$ CI: $0.27-0.72$, $p=0.001$ ), had history of TB treatment (OR: $1.76,95 \%$ CI: 1.15-2.69, $p=0.009)$, experienced anti-TB drug adverse effects (OR: $0.65,95 \%$ CI: $0.46-0.93, p=0.017$ ), experienced stigma (OR: $0.67,95 \%$ CI: $0.47-0.97, p=0.032$ ), or had supervised treatment (OR: $0.66,95 \%$ CI: $0.47-0.91, p=0.012)$ tended to perform lower medication adherence (Table 5).

\section{Discussion}

This study aimed to assess pulmonary TB patients' medication adherence and explore its determinants by assessing sociodemographic characteristics, treatment factors, knowledge about TB, mental health, and behavioral characteristics. The strengths of our study included the fact that it was conducted in northeast China, where few relevant studies have been conducted. Additionally, based on the bio-psycho-social medical model, we innovatively introduced treatment factors and perceived stigma into the analysis of medication adherence determinants in the Chinese context. The results showed that, overall, TB patients' medication adherence was not very high, and only $41.84 \%$ reported high adherence to their anti-TB medicine regimen. In addition, various factors were associated with medication adherence and it is imperative to explore the reasons and formulate feasible measures to improve medication adherence.

Table 5 Ordinal Logistic Regression Exploring Medication Adherence Among TB Patients

\begin{tabular}{|c|c|c|c|c|c|c|}
\hline \multirow{2}{*}{ Variable } & \multirow[t]{2}{*}{ Reference Category } & \multirow[t]{2}{*}{ Coefficient } & \multirow[t]{2}{*}{$p$-value } & \multirow[t]{2}{*}{ OR } & \multicolumn{2}{|c|}{$95 \% \mathrm{Cl}$ for $\mathrm{OR}$} \\
\hline & & & & & Low & High \\
\hline Age & - & 0.01 & 0.013 & 1.02 & 1.00 & 1.03 \\
\hline Unemployment & Yes & 0.48 & 0.011 & 1.61 & 1.12 & 2.32 \\
\hline Education & - & 0.10 & 0.305 & 1.11 & 0.91 & 1.34 \\
\hline History of TB treatment & Yes & 0.56 & 0.009 & 1.76 & 1.15 & 2.69 \\
\hline Medication duration & - & -0.12 & 0.090 & 0.89 & 0.77 & 1.02 \\
\hline Number of medicines & - & 0.05 & 0.379 & 1.05 & 0.94 & 1.18 \\
\hline Adjuvant drug use & Yes & -0.82 & 0.001 & 0.44 & 0.27 & 0.72 \\
\hline Adverse drug reaction & No & -0.42 & 0.017 & 0.65 & 0.46 & 0.93 \\
\hline Knowledge about TB & - & 0.29 & $<0.001$ & 1.34 & 1.16 & 1.54 \\
\hline Anxiety & - & -0.04 & 0.069 & 0.96 & 0.92 & 1.00 \\
\hline Stigma & No & -0.39 & 0.032 & 0.67 & 0.47 & 0.97 \\
\hline Self-assessed severity & - & 0.07 & 0.426 & 1.07 & 0.90 & 1.27 \\
\hline Smoking & Yes & 0.30 & 0.204 & 1.35 & 0.85 & 2.16 \\
\hline Alcohol consumption & Yes & $0.6 \mathrm{I}$ & 0.032 & 1.84 & 1.05 & 3.22 \\
\hline Medication supervision & Unsupervised & -0.42 & 0.012 & 0.66 & 0.47 & 0.91 \\
\hline
\end{tabular}

Note: "-" means not applicable.

Abbreviations: $\mathrm{TB}$, tuberculosis; $\mathrm{OR}$, odds ratio; $\mathrm{Cl}$, confidence interval. 
We found that medication adherence increased with age, which may be because the older patients exhibited more socially conscious behavior, and had more life experiences and stronger ideology that led them to adhere more strongly to their anti-TB medicine regimen. In a study in the USA, older latently infected TB patients exhibited a higher treatment completion rate than the younger patients. ${ }^{27}$ In contrast, younger TB patients may be more likely to be non-adherent because of various risk factors, ${ }^{28}$ so we should pay more attention to young patients and adopt enhanced adherence measures if necessary. ${ }^{29}$ In addition, we found that unemployed patients exhibited insufficient medication adherence. As a chronic disease, TB patients require extra money over the long term to purchase adjuvant drugs and health services in addition to the free treatment provided in China, and TB can cause a heavy financial burden on a patient's family if he or she is unemployed. ${ }^{30,32}$ Being employed not only contributes to resolving the problem of the financial burden of TB treatment, but it increases social participation, which may be beneficial to increase medication adherence. However, many Chinese employers discriminate against patients with infections, especially pulmonary $\mathrm{TB}$, and are unwilling to hire them. Therefore, the government should take patients' employment status into consideration when they formulate supportive policies to promote anti-TB medication adherence.

Several treatment factors, but not all, were significantly associated with medication adherence. Our study found that the relapse TB patients were more likely to show low medication adherence than the new diagnosed ones. The new TB patients may have stronger confidence and determination to cure the disease which facilitate them to take anti-tuberculosis medicine in time and quantity; however, the relapse patients may hold the idea that the disease would recur even it is cured temporarily and they may also require more complicated treatment, so they could take medicine not seriously. ${ }^{33,34}$ In a previous study, medication duration was significantly negatively associated with medication adherence, ${ }^{35}$ but the association in our study was not significant $(p=0.090)$, which may be due to regional differences. Additionally, the number of anti-TB drugs patients took and whether they only took free anti-TB drugs were not associated with medication adherence. Thus, these aspects of the medication regimen itself did not decrease adherence, but rather the other effects of the medication (adjuvant medicine use and adverse drug effects) did. In practice, doctors prescribe anti-TB drugs together with adjuvant medicine (such as hepatinica) to protect the patients' organs from damage, but a few patients in our study did not follow their doctors' advice to take adjuvant medicine. Refusal of adjuvant medicine was associated with lower medication adherence, which may be because: 1) some patients may express disgust at both anti-TB medicine and the adjuvant drugs when taking various medicines every day, ${ }^{36}$ 2) not taking adjuvant medicine would lead to more adverse drug reactions. Moreover, adverse drug reactions were associated with low medication adherence. If patients are not informed in advance about the adverse effects caused by anti-TB drugs by medical staff, patients can become fearful in response to adverse effects and even abandon treatment. ${ }^{37,38}$ Therefore, it is urgent to enhance patient-doctor communication regarding the effects of the adjuvant and anti-TB medicines' adverse effects to eliminate patients' misunderstandings about the treatment process.

Medication adherence significantly increased when participants had a good understanding of TB prevention and treatment. A case-control study conducted in Kenya also found that inadequate knowledge about TB was independently associated with low medication adherence. ${ }^{13}$ The current public education about TB prevention and treatment in China is insufficient. ${ }^{39}$ Most pulmonary TB patients can be cured if they take medicine regularly ${ }^{40}$ but, according to the present survey, some participants were unsure of whether the disease could be cured and some did not know the treatment duration. Therefore, comprehensive education and counseling at TB treatment initiation is important to improve medication adherence. ${ }^{38,41,42}$ Additionally, patients who perceived stigma exhibited significantly reduced medication adherence, which is consistent with a previous qualitative study conducted in South Africa. ${ }^{15}$ However, according to a case-control study in Korea, the absence of stigma was unexpectedly a predictor of low adherence. ${ }^{43}$ Stigma should not be thought of as a problem of patients themselves, but as a serious social issue in the current Chinese sociocultural context. ${ }^{26}$ Patients who experience stigma may be solitary, lacking assistance, and dispirited, which could affect their confidence in anti-TB treatment. Hence, it is necessary to reduce the social prejudice against $\mathrm{TB}$ patients and pay more attention to supplying psychological support to increase medication adherence.

Unhealthy habits were another important aspect of predicting medication adherence. Alcohol consumption was associated with low medication adherence. Alcohol 
consumption can paralyze nerves and cause unconsciousness, which could result in forgetting to take anti-TB medicine. $^{44,45}$ Moreover, both alcohol consumption and taking anti-TB medicine can cause liver damage, which could reduce the patients' motivation to take the medication due to experiencing body pain. ${ }^{46}$ In addition, we found that unsupervised treatment was associated with increased medication adherence. This may be interpreted as reflecting the fact that patients who managed their own treatment may be proactive and have increased acceptance of regularly taking anti-TB treatment, which manifested in increased medication adherence. In contrast, patients who need treatment supervision may be careless about their disease and reluctant to take their medicine. Of course, further specifically designed studies are needed to clarify the reasons for this result. Based on these findings, medical personnel should increase awareness about the harm of alcohol consumption during treatment, and valid measures should be implemented to enhance supervised treatment and patients' motivation to take treatment.

Several limitations in this study should be mentioned. The cross-sectional design could only indicate the current medication adherence of TB patients, so the associations observed in the study cannot be interpreted as causal associations between various statistically significant factors and medication adherence. Additionally, limitation regarding the sources of the sample may restrict the generalization of the study conclusions, as they may not be representative of other areas. Moreover, there could be reporting bias because the participants were required to self-report the relevant information. Finally, there was a lack of deep analysis of the mediation effects regarding medication adherence, so complex mechanisms influencing medication adherence were not explored in detail. Given these limitations, conducting future research on longitudinal and systematic (including medical) data is essential to effectively explore more determinants of medication adherence.

\section{Conclusion}

This study on the medication adherence of pulmonary TB patients was conducted in Dalian in northeast China, where there has previously been a lack of relevant research. Further, we explored the determinants of medication adherence based on the bio-psycho-social medical model. Tuberculosis patients' medication adherence was not very high. The factors influencing the medication adherence of TB patients are diverse and complex which involved sociodemographic characteristics, treatment factors, knowledge about TB, mental health, and behavioral characteristics. Patients who were older were employed, had more knowledge about $\mathrm{TB}$, and did not consume alcohol were more likely to have increased medication adherence. However, those who did not follow their doctors' advice to take adjuvant drugs, experienced anti-TB drug adverse effects, had a history of TB treatment, experienced perceived stigma, and had supervised treatment tended to exhibit lower medication adherence during treatment. Therefore, in particular, social support, sufficient health education and counseling, indispensable alcohol control and medication supervision interventions are recommended to effectively improve medication adherence.

\section{Acknowledgments}

We thank all 15 interviewers, who came from the School of Public Health at Dalian Medical University, for their efforts in collecting the data. In addition, we are grateful to all the medical personnel from the four investigative districts who contributed to our study. Importantly, we express gratitude to the TB patients who made our study possible. Finally, we thank Dr. Morisky for allowing us to use MMAS- 8 , and the use of the CMMAS is protected by US copyright and registered trademark laws. Permission for use is required. A license agreement is available from: Donald E. Morisky, 294 Lindura Court, Las Vegas, NV 89138-4632; dmorisky@gmail.com.

\section{Author Contributions}

All authors contributed to data collection and analysis, drafted and revised the manuscript, gave final approval to publish the manuscript, and agreed to be accountable for all aspects of the work.

\section{Funding}

This research received no external funding.

\section{Disclosure}

The authors report no conflicts of interest in this work.

\section{References}

1. World Health Organization. Global tuberculosis report 2019. Geneva, Switzerland: World Health Organization;2019. WHO/CDS/TB/201 9.15 .

2. Harper I. Extreme condition, extreme measures? Compliance, drug resistance, and the control of tuberculosis. Anthropol Med. 2010;17 (2):201-214. doi:10.1080/13648470.2010.493606 
3. $\mathrm{Xu} \mathrm{CH}$, Jeyashree $\mathrm{K}$, Shewade $\mathrm{HD}$, et al. Inequity in catastrophic costs among tuberculosis-affected households in China. Infect Dis Poverty. 2019;8(1):46. doi:10.1186/s40249-019-0564-2

4. Menzies NA, Gomez GB, Bozzani F, et al. Cost-effectiveness and resource implications of aggressive action on tuberculosis in China, India, and South Africa: a combined analysis of nine models. Lancet Glob Health 2016;4(11):e816-e826. doi:10.1016/S2214-109X(16)30265-0

5. Mekonnen HS, Azagew AW. Non-adherence to anti-tuberculosis treatment, reasons and associated factors among TB patients attending at Gondar town health centers, Northwest Ethiopia. BMC Res Notes. 2018;11(1):691. doi:10.1186/s13104-018-3789-4

6. Adane AA, Alene KA, Koye DN, Zeleke BM. Non-adherence to anti-tuberculosis treatment and determinant factors among patients with tuberculosis in Northwest Ethiopia. PLoS One. 2013;8(11):6. doi:10.1371/journal.pone.0078791

7. Martinez L, Shen Y, Mupere E, Kizza A, Hill PC, Whalen CC Transmission of Mycobacterium tuberculosis in households and the community: a systematic review and meta-analysis. Am J Epidemiol. 2017;185(12):1327-1339. doi:10.1093/aje/kwx025

8. Yao S, Huang WH, van den Hof S, et al. Treatment adherence among sputum smear-positive pulmonary tuberculosis patients in mountainous areas in China. BMC Health Serv Res. 2011;11(1):341. doi:10.1186/1472-6963-11-341

9. Lei X, Huang K, Liu Q, Jie YF, Tang SL. Are tuberculosis patients adherent to prescribed treatments in China? Results of a prospective cohort study. Infect Dis Poverty. 2016;5(1):38. doi:10.1186/s40249-016-0134-9

10. Zhou C, Chu J, Liu J, et al. Adherence to tuberculosis treatment among migrant pulmonary tuberculosis patients in Shandong, China: a quantitative survey study. PLoS One. 2012;7(12):e52334. doi:10.1371/journal.pone.0052334

11. Jakubowiak WM, Bogorodskaya EM, Borisov SE, Danilova ID, Lomakina OB, Kourbatova EV. Impact of socio-psychological factors on treatment adherence of TB patients in Russia. Tuberculosis (Edinb). 2008;88(5):495-502. doi:10.1016/j.tube.2008.03.004

12. Janakan N, Seneviratne R. Factors contributing to medication noncompliance of newly diagnosed smear-positive pulmonary tuberculosis patients in the district of Colombo, Sri Lanka. Asia Pac J Public Health. 2008;20(3):214-223. doi:10.1177/1010539508316974

13. Muture BN, Keraka MN, Kimuu PK, Kabiru EW, Ombeka VO, Oguya F. Factors associated with default from treatment among tuberculosis patients in Nairobi province, Kenya: a case control study. BMC Public Health. 2011;11(1):696. doi:10.1186/1471-2458-11-696

14. Theron G, Peter J, Zijenah L, et al. Psychological distress and its relationship with non-adherence to TB treatment: a multicentre study. BMC Infect Dis. 2015;15(1):253. doi:10.1186/s12879-015-0964-2

15. Skinner D, Claassens M. It's complicated: why do tuberculosis patients not initiate or stay adherent to treatment? A qualitative study from South Africa. BMC Infect Dis. 2016;16(1):712. doi:10.1186/s12879-016-2054-5

16. Munro SA, Lewin SA, Smith HJ, Engel ME, Fretheim A, Volmink J. Patient adherence to tuberculosis treatment: a systematic review of qualitative research. PLoS Med. 2007;4(7):e238. doi:10.1371/journal. pmed.0040238

17. Tang Y, Zhao M, Wang Y, et al. Non-adherence to anti-tuberculosis treatment among internal migrants with pulmonary tuberculosis in Shenzhen, China: a cross-sectional study. BMC Public Health. 2015;15(1):474. doi:10.1186/s12889-015-1789-Z

18. Engel GL. The need for a new medical model: a challenge for biomedicine. Science. 1977;196(4286):129-136. doi:10.1126/ science. 847460

19. Engel GL. The clinical application of the biopsychosocial model. $A m$ J Psychiatry. 1980;137(5):535-544.

20. Ogundele OA, Moodley D, Pillay AW, Seebregts CJ. An ontology for factors affecting tuberculosis treatment adherence behavior in sub-Saharan Africa. Patient Prefer Adherence. 2016;10:669-681. doi:10.2147/PPA.S96241
21. Morisky DE, Ang A, Krousel-Wood M, Ward HJ. Predictive validity of a medication adherence measure in an outpatient setting. J Clin Hypertens (Greenwich). 2008;10(5):348-354. doi:10.1111/j.17517176.2008.07572.x

22. Valencia S, Leon M, Losada I, Sequera VG, Fernandez Quevedo M, Garcia-Basteiro AL. How do we measure adherence to anti-tuberculosis treatment? Expert Rev Anti Infect Ther. 2017;15 (2):157-165. doi:10.1080/14787210.2017.1264270

23. Bress AP, Bellows BK, King JB, et al. Cost-effectiveness of intensive versus standard blood-pressure control. N Engl J Med. 2017;377 (8):745-755. doi:10.1056/NEJMsa1616035

24. Berlowitz DR, Foy CG, Kazis LE, et al. Effect of intensive blood-pressure treatment on patient-reported outcomes. $N$ Engl J Med. 2017;377(8):733-744. doi:10.1056/NEJMoa1611179

25. Spitzer RL, Kroenke K, Williams JB, Lowe B. A brief measure for assessing generalized anxiety disorder: the GAD-7. Arch Intern Med. 2006;166(10):1092-1097. doi:10.1001/archinte.166.10.1092

26. Xu M, Markstrom U, Lyu J, Xu L. Survey on tuberculosis patients in rural areas in China: tracing the role of stigma in psychological distress. Int J Environ Res Public Health. 2017;14(10):10. doi:10.33 90/ijerph14101171

27. Li J, Munsiff SS, Tarantino T, Dorsinville M. Adherence to treatment of latent tuberculosis infection in a clinical population in New York City. Int J Infect Dis. 2010;14(4):e292-297. doi:10.1016/j.ijid.20 09.05 .007

28. Guix-Comellas EM, Rozas-Quesada L, Force-Sanmartin E, EstradaMasllorens JM, Galimany-Masclans J, Noguera-Julian A. Influence of nursing interventions on adherence to treatment with antituberculosis drugs in children and young people: research protocol. $J A d v$ Nurs. 2015;71(9):2189-2199. doi:10.1111/jan.12656

29. Costa E, Giardini A, Savin M, et al. Interventional tools to improve medication adherence: review of literature. Patient Prefer Adherence. 2015;9:1303-1314. doi:10.2147/PPA.S87551

30. Xin YJ, Xiang L, Jiang JN, Lucas H, Tang SL, Huang F. The impact of increased reimbursement rates under the new cooperative medical scheme on the financial burden of tuberculosis patients. Infect Dis Poverty. 2019;8(1):9. doi:10.1186/s40249-019-0575-z

31. Duan WX, Zhang W, Wu CG, et al. Extent and determinants of catastrophic health expenditure for tuberculosis care in Chongqing municipality, China: a cross-sectional study. BMJ Open. 2019;9 (4):10. doi:10.1136/bmjopen-2018-026638

32. Mao WH, Jiang WX, Hamilton C, et al. Over- and under-treatment of TB patients in Eastern China: an analysis based on health insurance claims data. Trop Med Int Health. 2019;24(9):1078-1087. doi:10.11 11/tmi.13287

33. Lukoye D, Adatu F, Musisi K, et al. Anti-tuberculosis drug resistance among new and previously treated sputum smear-positive tuberculosis patients in Uganda: results of the first national survey. PLoS One. 2013;8(8):e70763. doi:10.1371/journal.pone.0070763

34. Wang $\mathrm{K}$, Chen S, Wang X, et al. Factors contributing to the high prevalence of multidrug-resistant tuberculosis among previously treated patients: a case-control study from China. Microb Drug Resist. 2014;20(4):294-300. doi:10.1089/mdr.2013.0145

35. Park S, Sentissi I, Gil SJ, et al. Medication event monitoring system for infectious tuberculosis treatment in Morocco: a retrospective cohort study. Int J Environ Res Public Health. 2019;16(3):412.

36. Williams G, Alarcon E, Jittimanee S, et al. Guidance for the implementation of best practice for the care of patients with tuberculosis. Int J Tuberc Lung Dis. 2008;12(3):236-240.

37. Hasker E, Khodjikhanov M, Sayfiddinova S, et al. Why do tuberculosis patients default in Tashkent City, Uzbekistan? A qualitative study. Int J Tuberc Lung Dis. 2010;14(9):1132-1139.

38. M'Imunya JM, Kredo T, Volmink J. Patient education and counselling for promoting adherence to treatment for tuberculosis. Cochrane Database Syst Rev. 2012;(5). doi:10.1002/14651858.CD006591. pub2. 
39. Li Y, Zhang S, Zhang T, et al. Chinese health literacy scale for tuberculosis patients: a study on development and psychometric testing. BMC Infect Dis. 2019;19(1):545. doi:10.1186/s12879-019-41 $68-\mathrm{z}$

40. Lonnroth K, Castro KG, Chakaya JM, et al. Tuberculosis control and elimination 2010-50: cure, care, and social development. Lancet. 2010;375(9728):1814-1829. doi:10.1016/S0140-6736(10) 60483-7

41. Nieuwlaat R, Wilczynski N, Navarro $\mathrm{T}$, et al. Interventions for enhancing medication adherence (Review). Cochrane Database Syst Rev. 2014;(11):CD000011.

42. Widjanarko B, Gompelman M, Dijkers M, van der Werf MJ. Factors that influence treatment adherence of tuberculosis patients living in Java, Indonesia. Patient Prefer Adherence. 2009;3:231-238. doi:10. 2147/ppa.s6020
43. Park CK, Shin HJ, Kim YI, et al. Predictors of default from treatment for tuberculosis: a single center case-control study in Korea. J Korean Med Sci. 2016;31(2):254-260. doi:10.3346/jkms.2016.31.2.254

44. Moro RN, Borisov AS, Saukkonen J, et al. Factors associated with noncompletion of latent tuberculosis infection treatment: experience from the PREVENT TB trial in the United States and Canada. Clin Infect Dis. 2016;62(11):1390-1400. doi:10.1093/cid/ciw126

45. Peltzer K, Naidoo P, Matseke G, Louw J, McHunu G, Tutshana B. Prevalence of psychological distress and associated factors in tuberculosis patients in public primary care clinics in South Africa. BMC Psychiatry. 2012;12(1). doi:10.1186/1471-244X-12-89

46. Guo YX, Xu XF, Zhang QZ, et al. The inhibition of hepatic bile acids transporters Ntcp and Bsep is involved in the pathogenesis of isoniazid/rifampicin-induced hepatotoxicity. Toxicol Mech Methods. 2015;25(5):382-387. doi:10.3109/15376516.2015.1033074

\section{Publish your work in this journal}

Patient Preference and Adherence is an international, peer-reviewed, open access journal that focuses on the growing importance of patient preference and adherence throughout the therapeutic continuum. Patient satisfaction, acceptability, quality of life, compliance, persistence and their role in developing new therapeutic modalities and compounds to optimize clinical outcomes for existing disease states are major areas of interest for the journal. This journal has been accepted for indexing on PubMed Central. The manuscript management system is completely online and includes a very quick and fair peer-review system, which is all easy to use. Visit http:// www.dovepress.com/testimonials.php to read real quotes from published authors. 